\title{
1-D finite volume model for dam-break induced mud-flow
}

\author{
L. Schippa \& S. Pavan \\ Department of Engineering, Ferrara University, Italy
}

\begin{abstract}
A numerical model suitable for the simulation of dam break flow, for Newtonian or non-Newtonian fluids, has been developed. The main features of the model are concerned with the propagation of wet-dry fronts, the treatment of irregular and variable cross sections shape, and the applicability to highly sloping channels. Governing equations are numerically solved using a simple first-order finite volume scheme. Different test cases have been selected in order to check all the fundamental features necessary for debris and mud-flow simulation. The model has finally been tested using laboratory experiments on mud-flow dambreak over a sloping plane. Numerical results compare favourably with experiments in terms of front wave speed, peak height and residual front thickness.
\end{abstract}

Keywords: mud-flow, dam-break, source terms, numerical model.

\section{Introduction}

The aim of the present work is the setting-up of a numerical model suitable for the simulation of mud-flows in channels of complex geometry. To fulfil this purpose, the model should have specific features that will be examined in detail, such as the treatment of wet-dry fronts, the handling of complex geometries and high bed slopes and the possibility of changing the model application field from Newtonian to non-Newtonian fluids, simply by changing the resistance law.

The proposed model is based on an alternative formulation of conservative balance equations, which includes a particular mathematical expression of source terms ideated for natural channels, and which has already demonstrated important stability features under the numerical point of view $[1,2]$. This formulation is kept in the present work, and the innovation stands in the numerical implementation, since a finite volume method based on Roe's scheme 
is used instead of the MacCormack finite difference method [1, 2]. The main reason is the necessity of correctly capturing front wave propagation speed in case of initially dry bed, for which Riemann-solver based techniques are recommended $[3,4]$. The second reason is the intention to verify if the model stability features are kept also if the numerical implementation radically changes.

Finite volume schemes are largely diffused in mud-flows treatment [5-7], and the Roe's approximation is often the chosen Riemann solver technique. The presented model uses the same approach, but paying careful attention in conserving the general formulation for complex geometry channels. This results in a particular expression of the wave propagation celerity, which does not depend on water depth or cross-section width. These hydraulic quantities are often corrected or mediated to be representative of irregular cross-sections, or, on the other hand, cross-section shape may be parameterized to be numerically handled [8]. In this work celerity is determined referring to cross section wetted area and static moment, in order to ensure the formulation generality.

For what concerns the source terms treatment, one should distinguish between pressure terms and friction terms. The pressure source terms, induced by the channel irregular geometry have been treated as in [1, 2], mathematically transforming the derivative of the static moment in order to eliminate the explicit dependence from the channel bed slope. This operation keeps its validity also in case of highly sloping channels, condition which often occurs in mud-flow phenomena. Friction source terms depends on the evaluation of friction slope, and therefore on the adopted resistance law. Like most of numerical models [6], the proposed model set up permits to easily change the resistance law and therefore to use the best fitting rheological model for each test case. Finally, source terms numerical implementation has been kept as simple as possible, to put in evidence the stability features coming from the basis mathematical model. They are therefore handled using the splitting technique [3] and computed with the Euler's method.

All the mentioned features have been investigated choosing specific test cases. The classic frictionless dam-break test is used to verify the correctness of waves speed propagation and the capability of treating wet-dry fronts. A noncylindrical frictionless ideal channel is used to evaluate the model response to abrupt changes in cross-sections wideness and bed elevation. The effect of friction terms introduction is then checked using a mud flow dam-break, for which the analytical solution is available. This test permits to consider and compare different resistance formulas, and can therefore also be used as a proof field for the introduction of different rheological schemes inside the model. It is also useful for testing the wave front propagation speed and the liquid-solid discharge stopping conditions. The model has further been tested using laboratory experiments on mud flow dam-break over a sloping plane.

\section{Mathematical model}

The mathematical model is based on an alternative formulation of balance equations for 1-D flows in natural channels of complex geometry [1]. The 
continuity equation and the momentum balance equation are written in terms of state variables $A$ and $Q$, considering no lateral inflows.

$$
\begin{gathered}
\frac{\partial A}{\partial t}+\frac{\partial Q}{\partial x}=0 \\
\frac{\partial Q}{\partial t}+\frac{\partial}{\partial x}\left(\frac{Q^{2}}{A}+g I_{1}\right)=\left.g \frac{\partial I_{1}}{\partial x}\right|_{z_{w}}-g A S_{f}
\end{gathered}
$$

where $t$ is time, $x$ is distance along the channel, $A$ the wetted cross-sectional area, $Q$ the discharge, $g$ the gravitational acceleration, $I_{1}$ the static moment of the wetted area, defined as:

$$
I_{1}=\cos \vartheta \int_{0}^{h(x)} b(x, z)(h(x)-z) \mathrm{d} z
$$

$I_{2}$ is the variation of the static moment $I_{1}$ along the $x$-direction, $S_{o}=\sin \vartheta$, where $\vartheta$ is the angle between channel bottom and the horizontal, $b$ is the cross-section width, $h$ is flow depth.

The system closure equation for the evaluation of the friction term $S_{f}$ will be described in detail for each examined test case, but the generally considered formulation is

$$
S_{f}=\frac{\tau}{\rho g R}
$$

in which $S_{f}$ is the slope friction, $R$ is the hydraulic radius, $\rho$ is the mixture or the fluid density, and the shear stress $\tau$ depends on the adopted rheological model.

\subsection{The source term}

Differently from the commonly used formulation of shallow water equations, the proposed model does not include in the momentum balance equation source term a direct dependence on bed slope. Details on the mathematical treatment which led to eqn. (2) can be found in [2].

The classic momentum equation is

$$
\frac{\partial Q}{\partial t}+\frac{\partial}{\partial x}\left(\frac{Q^{2}}{A}+g I_{1}\right)=g A\left(S_{0}-S_{f}\right)+g I_{2}
$$

Focusing on the source term, the pressure term $I_{2}$ has the following expression:

$$
I_{2}=\left.\frac{\partial I_{1}}{\partial x}\right|_{h}=\cos \vartheta \int_{0}^{h(x)}(h(x)-z) \frac{\partial b(x, z)}{\partial x} \mathrm{~d} z
$$

Briefly, the pressure term $I_{2}$ can be expressed as the sum of two terms, one of which is the variation of static moment $I_{1}$ along $x$ considering the water surface elevation $z_{w}$ as a constant, while the other exactly balances gravitational forces in the momentum equation, unless the presence of the term $\cos \vartheta$ which arises in 
case of high slopes, and cannot be neglected when considering mud-flow or debris-flow phenomena.

$$
I_{2}=\left.\frac{\partial I_{1}}{\partial x}\right|_{z_{w}}-S_{0} A \cos \vartheta
$$

The substitution into (6) produces:

$$
g A\left(S_{0}-S_{f}\right)+g I_{2}=g A S_{0}(1-\cos \vartheta)-g A S_{f}+\left.g \frac{\partial I_{1}}{\partial x}\right|_{z_{w}}
$$

In this case, the term $A S_{0}$ does not disappear as illustrated in [1] and [2], but it remains and it is multiplied by the factor $(1-\cos \vartheta)$. However, numerical proofs have demonstrated that this term is little if compared to friction terms, and can therefore be neglected. Eqn. (2) is therefore valid also for high sloping channel and debris flow simulation.

\section{Numerical model}

Shallow water equations have been numerically implemented using the firstorder finite volumes Godunov scheme. Numerical fluxes are computed with Roe's method and source terms are evaluated with Euler's approach and taken into account adopting the splitting technique. Details on the different components of the numerical model can be found in Toro [3]. The resultant scheme is explicit, first-order accurate, and has a very uncomplicated structure, since it is built choosing the simplest solution technique for every element of the partial differential equations system. This approach has the intention to illustrate the intrinsic stability features of the mathematical model, which could otherwise be hidden by sophisticated numerical schemes.

Referring to shallow water equations in the vector form (eqn. (9)) the splitting approach for source terms treating, consists in separately solving the homogeneous partial differential equations system (eqn. (10)) and the ordinary differential equation (eqn. (11)). In detail, the solution obtained from eqn. (10) is used as initial condition for eqn. (11).

$$
\begin{gathered}
\mathbf{U}_{t}+\mathbf{F}(\mathbf{U})_{x}=\mathbf{S}(\mathbf{U}) \\
\mathbf{U}_{t}+\mathbf{F}(\mathbf{U})_{x}=0 \Rightarrow \overline{\mathbf{U}} \\
\mathbf{U}_{t}=\mathbf{S}(\overline{\mathbf{U}}) \Rightarrow \mathbf{U}_{t+d t}
\end{gathered}
$$

The Roe's scheme, used to solve eqn. (6), requires the definition of the Jacobian matrix

$$
\mathbf{J}=\frac{\partial \mathbf{F}}{\partial \mathbf{U}}=\left(\begin{array}{cc}
0 & 1 \\
g \frac{\partial I_{1}}{\partial A}-\frac{Q^{2}}{A^{2}} & 2 \frac{Q}{A}
\end{array}\right)=\left(\begin{array}{cc}
0 & 1 \\
c^{2}-u^{2} & 2 u
\end{array}\right)
$$


Most of models proposed in the literature about the resolution of shallow water equations for debris flow or natural channels, based on approximate Riemann solvers (see for example $[4,5,9]$ ), adopt the same simplification in the evaluation of the term $\partial I_{1} / \partial A$, assuming

$$
\frac{\partial I_{1}}{\partial A} \simeq \frac{A}{B} \simeq h \Rightarrow c=\sqrt{g \frac{A}{b}} \quad \text { or } \quad c=\sqrt{g h}
$$

In the present model, in order to keep the formulation generality and to ensure the applicability to natural and complex channel geometries, the static moment derivative is explicitly computed as the variation of $I_{1}$ relative to the variation of $A$ in the water depth variation range $h \pm \Delta h$

$$
\frac{\partial I_{1}}{\partial A}=\frac{I_{1}(h+\Delta h)-I_{1}(h-\Delta h)}{A(h+\Delta h)-A(h-\Delta h)}
$$

The celerity $c$ is therefore defined as

$$
c=\sqrt{g \frac{\partial I_{1}}{\partial A}}
$$

Another important aspect of Godunov finite volume method application to natural geometries, is the quantification of cell water volume $V$ and the definition of the relation between the state variable $A$ and $V$. For every computational cell, $A$ is defined as

$$
A_{i}=\frac{1}{\Delta x} \int_{x_{i-\frac{1}{2}}}^{x_{i+\frac{1}{2}}} A(x, t) \mathrm{d} x=\frac{V_{i}}{\Delta x}
$$

$V_{i}$ is computed as the volume of a pyramid which bases are irregular polygons, since the water profile is assumed to be parallel to channel bed.

$$
V_{i}=\frac{\left(A_{i-\frac{1}{2}}+A_{i+\frac{1}{2}}+\sqrt{A_{i-\frac{1}{2}} A_{i+\frac{1}{2}}}\right) \Delta x}{3}
$$

\subsection{Source terms numerical treatment}

Source terms are numerically included in computations by splitting, and they are simply computed by Euler's method

$$
\mathbf{U}_{t+d t}=\mathbf{U}_{t}+\Delta t \cdot \mathbf{S}(t, \overline{\mathbf{U}})
$$

in which

$$
\mathbf{S}=\left(\begin{array}{c}
0 \\
\left.g \frac{\partial I_{1}}{\partial x}\right|_{z_{w}}-g A S_{f}
\end{array}\right)
$$



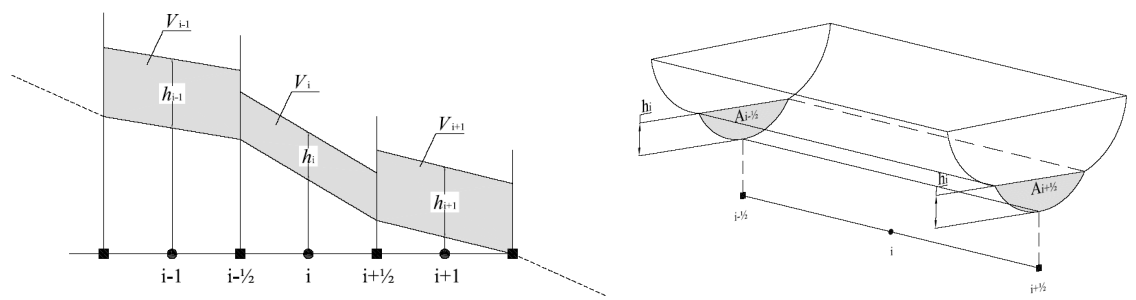

Figure 1: $\quad$ Computational scheme for $V_{i}$.

Considering no lateral inflows, source terms are present only in the momentum balance equation. This term can be divided into two parts, that is the friction term and the pressure term, represented by the static moment variation along channel, taking the water surface elevation as a constant.

The computational scheme for the pressure term quantification in represented in Figure 2, and the variation of $I_{1}$ is computed as:

$$
\left.\left.\frac{\partial I_{1}}{\partial x}\right|_{z_{w}} \simeq \frac{\Delta I_{1}}{\Delta x}\right|_{z_{w}}=\frac{I_{1}\left(h_{i+\frac{1}{2}}\right)-I_{1}\left(h_{i-\frac{1}{2}}\right)}{\Delta x}
$$

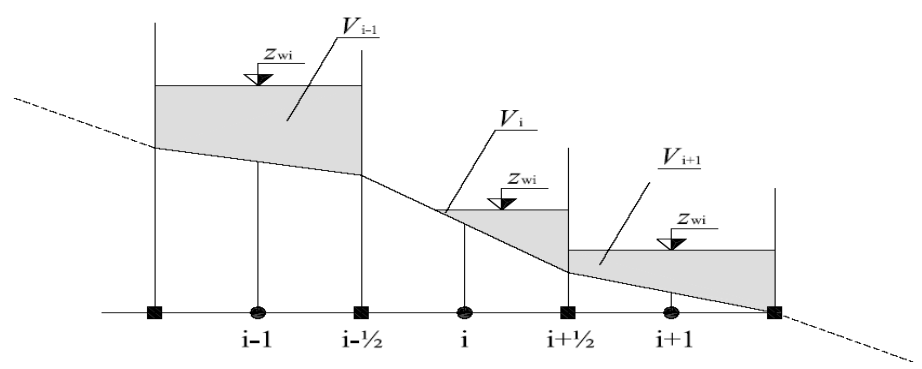

Figure 2: Computational scheme for momentum balance pressure source term.

\section{Numerical tests}

Five test cases have been selected to investigate the performances of the proposed mathematical model and numerical scheme. Test cases 1 and 2 are idealized dam-break problems in a rectangular channel with dry bed and wet bed respectively. These examples have been chosen in order to check the model capability to address wet-dry fronts and to compute the correct wave speed propagation. The $3^{\text {rd }}$ test case proposes the simulation of water at rest inside a non-cylindrical frictionless idealized channel, and is used to check model response to geometrical and pressure source terms. With test case 4 the attention is shifted to non-Newtonian fluids, with a mud-flow dam break over horizontal 
bed. In this test case the aim is to verify the correctness of the rheological model and the role of friction source terms. Finally test case 5 refers to laboratory experiments about mud-flow dam-break over a sloping plane.

\subsection{Idealized dam-break problem in a rectangular channel with dry bed}

Consider a $10 \mathrm{~m}$ long rectangular channel, with horizontal and frictionless bed. A dam is located in the middle, $5 \mathrm{~m}$ from upstream and downstream ends. Water depth upstream the dam is $1.5 \mathrm{~m}$, while downstream the bed is dry. In the simulations, $\Delta x=0.1 \mathrm{~m}$ is set, while $\Delta t$ is determined by the Courant-FriedrichsLewy stability condition assuming a CFL number equal to 0.9 .

In Figure 3(a), comparison between the analytical and numerical solution is shown at time $t=0.7 \mathrm{~s}$. The exact solution has been taken from Toro [3]. Wave front velocity is slightly under-predicted by the numerical scheme, but it is coherent with the adopted numerical scheme [3]. The use of the partial derivative of the static moment $I_{1}$ with respect to wetted area $A$ (eqn. (15)) in the definition of the celerity, instead of the water depth $h$, does not affect therefore the efficiency of the numerical scheme. The water profile discontinuity at $x=5 \mathrm{~m}$ is related to the need of an entropy fix correction for the Roe's approximate Riemann solver. In this case the Harten-Hyman entropy fix has been used.
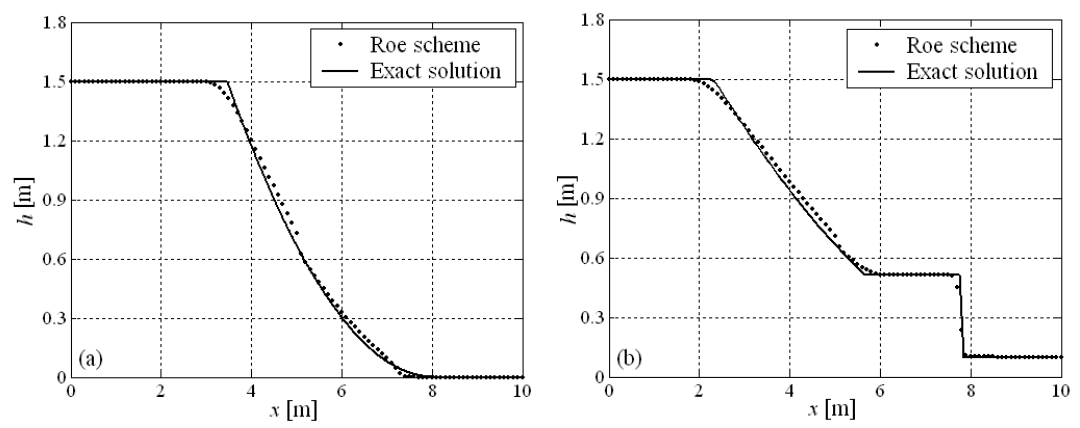

Figure 3: Comparison between analytical and numerical solution for the dam-break problem over dry bed (a) and over wet bed (b).

\subsection{Idealized dam-break problem in a rectangular channel with wet bed}

The geometry is the same as in the previous test case, but the downstream water depth is set to $0.1 \mathrm{~m}$. In this case the element to check is the propagation velocity of the downstream shock wave. In Figure 3(b) comparison between the analytical and numerical solution is shown at time $t=0.4 \mathrm{~s}$. The essential features of the flow, such as front location, shock wave height and front speed are well captured. As it is expected from the adopted first-order scheme, a light diffusivity is present in correspondence to solution discontinuities. The same entropy fix correction as in the previous test case has been added to reduce water profile discontinuity at $x=5 \mathrm{~m}$. 


\subsection{Non-cylindrical rectangular channel}

This test case, proposed by Goutal and Maurel [10], presents an hypothetical frictionless channel, which wideness and bottom elevation vary discontinuously along the longitudinal profile. Every cross section is rectangular in shape. As shown in Figure 4(a) and (b), this test presents abrupt bottom slope variations and sudden bank narrowing or widening, in particular at station $x=800 \mathrm{~m}$, channel breadth changes rapidly from 40 to 5 metres. The geometry is completely described by 29 cross sections. The total length is $1500 \mathrm{~m}$, and it has been divided into 1500 computational cells, assuming a constant cell dimension of $1 \mathrm{~m}$. The simulated flow condition is water at rest. As boundary conditions, zero discharge is imposed upstream $\left(Q_{M}=0 \mathrm{~m}^{3} / \mathrm{s}\right)$ and a constant level $z_{w V}=$ $12 \mathrm{~m}$ is kept downstream. The initial condition is still water with constant level $z_{w 0}=12 \mathrm{~m}$ and zero discharge $Q_{0}=0 \mathrm{~m}^{3} / \mathrm{s}$ at every cross section. Computation has been performed accounting for Courant-Friedrichs-Lewy stability condition, using a Courant number of 0.85 .
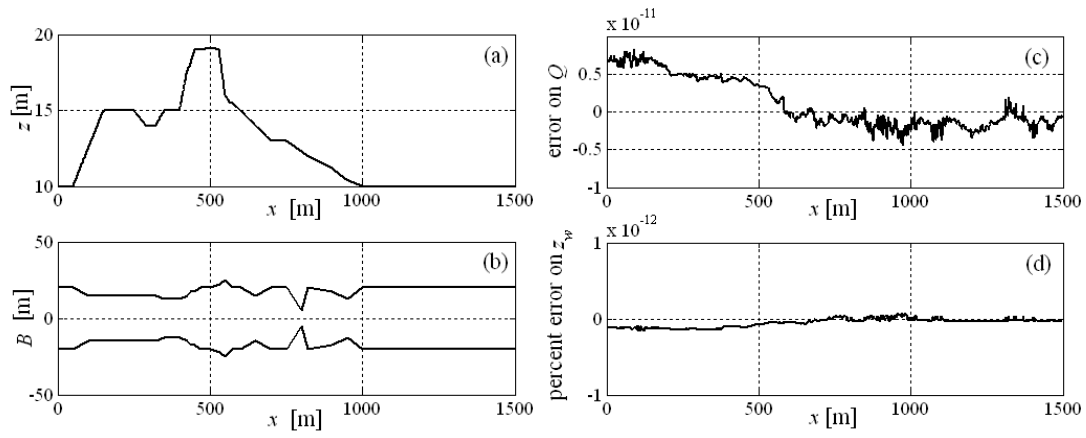

Figure 4: Non-cylindrical rectangular channel test: bed profile (a), channel plan (b), and numerical simulation errors on $Q$ (c) and on $z_{w}(\mathrm{~d})$.

The same test case has been treated in an authors' previous work [1] in which the proposed mathematical model has been implemented with the McCormack numerical scheme, obtaining for both discharge and water surface elevation a computational precision of $10^{-14}$. The application of the first order Roe's scheme leads to computational errors around $10^{-11}$, but it can however be considered as an encouraging result if compared with the Garcia-Navarro and Vazquez-Cendon [4] model, in which the Roe's approximate solver and the finite volume numerical scheme were applied in association with another source terms treatment. The comparison with the results reported in [4] highlights the role of the proposed pressure source terms mathematical treatment.

\subsection{Mud-low dam-break on an horizontal bed}

The fourth test case has the double function to shift the attention from Newtonian to non-Newtonian fluids and to verify the insertion of friction source terms 
inside the numerical model. For these purposes, the Hungr [11] test case ha $\mathrm{s}$ been selected. Hungr [11] gives a solution for a plastic fluid based on an approximated energy solution for a horizontal dam break problem consisting of a horizontal bed $1500 \mathrm{~m}$ long, with the dam positioned at $x=305 \mathrm{~m}$ from upstream. The initial water depth upstream the dam is $30.5 \mathrm{~m}$, while downstream the bed is dry. The stopping location for the water-solid mixture results at $x=$ $1896 \mathrm{~m}$ under Hungr's assumption for yield stress and density. This test is usually employed to compare different flow resistance relations, since it furnishes an analytical reference solution [7]. In this work, three rheological models have been implemented and inserted into the model, which formulation and parameters are summarized in Table 1 .

Table 1: $\quad$ Flow resistance relations and parameters.

\begin{tabular}{lll}
\hline Resistance law & Formulation & Parameters \\
\hline Full Bingham & $S_{f}=\frac{\tau_{0}}{\rho g h}$ with $\tau_{0}$ from: & $\mu_{B}=100 \mathrm{~Pa} \cdot \mathrm{s}$, \\
& $2 \tau_{y}{ }^{3}-3\left(\tau_{y}+2 \frac{\mu_{B} q}{h^{2}}\right) \tau_{0}{ }^{2}+\tau_{y}{ }^{3}=0$ & $\rho=0 \mathrm{~N} / \mathrm{m}^{2}$ \\
& $S_{f}=\frac{\tau_{0}}{\rho g h}$ with: & $\mu_{B}=1035 \mathrm{~kg} / \mathrm{m}^{3}$ \\
\hline Simplified Bingham & $\tau_{y}=1500 \mathrm{~N} \cdot \mathrm{s}$, \\
& $\tau_{0}=1.5 \tau_{y}+3 \frac{\mu_{B} q}{h^{2}}$ & $\rho=1835 \mathrm{~kg} / \mathrm{m}^{3}$ \\
\hline Turbulent, & $S f=\frac{n^{2} q \sqrt{q^{2}}}{h^{2} h_{r}^{4 / 3}}+\frac{\tau_{i}}{\rho g h}$ with: & $n=0.0667 \mathrm{~s} / \mathrm{m}^{1 / 3}$ \\
Coulomb \& Yield & $\delta=10^{\circ}$ \\
& $\tau_{i}=\min \left(\tau_{y} ; \rho g h \cos \vartheta \tan \delta\right)$ & $\tau_{y}=500 \mathrm{~N} / \mathrm{m}^{2}$ \\
& & $\rho=1835 \mathrm{~kg} / \mathrm{m}^{3}$ \\
\hline
\end{tabular}

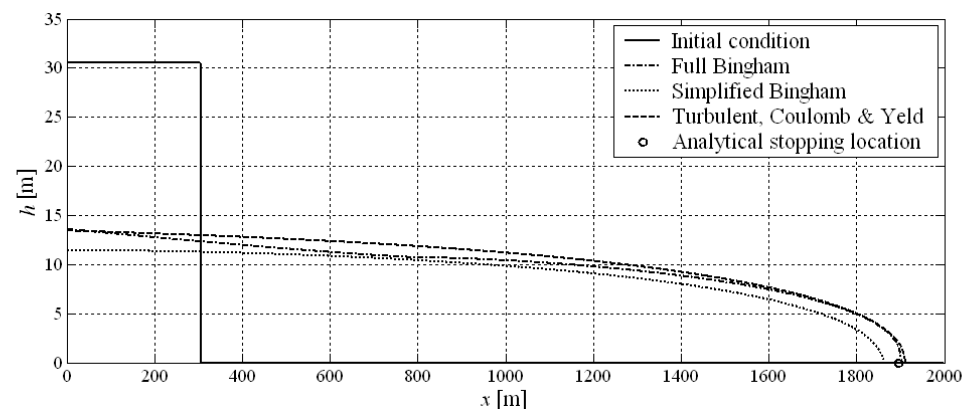

Figure 5: Mud-flow dam-break results for different resistance formulae.

For numerical simulations the cannel has been divided into 1500 cells of constant width. Results are depicted in Figure 5, and show in general a good behaviour of each resistance law. Apart from slight differences in the final flow profile, the analytical stopping position is satisfactorily approached. The aim of 
this test case however does not lay in the specific verification of different rheological models, but in the general model response to their implementation, and it can therefore be considered as fulfilled. Another important confirmation is model flexibility, since various flow resistance can be indifferently used. In further testing there will be therefore the possibility of choosing the best fitting rheological model.

\subsection{Mud-flow dam-break on a sloping plane}

In this test case numerical results are compared with the experimental results of Laigle and Coussot [7]. The experimental device consisted of a $4 \mathrm{~m}$ long and 0.6 $\mathrm{m}$ wide flume, in which a sluice, positioned at $0.85 \mathrm{~m}$ from the upstream end was rapidly pulled up reproducing a quasi-instantaneous dam-break for the solidliquid mixture. Flow depths were measured by three ultrasonic gauges positioned at $1.65,2.75$ and $3.85 \mathrm{~m}$ from upstream during the experiments. Several tests have been performed changing the plane slope form 6 to $31 \%$, and the simulated case has a $16 \%$ slope. The mixture had a measured density $\rho=1410 \mathrm{~kg} / \mathrm{m}^{3}$ and yield stress $\tau_{\mathrm{y}}=19 \mathrm{~Pa}$. The rheological model adopted is the same proposed by Laigle and Coussot [7] in their work. Mixture behaviour can in fact be described by Herschel-Bulkley model, which, for simple shear conditions may be written as:

$$
\tau=\tau_{c}+K \gamma^{\eta}
$$

Table 2: Comparison between experimental and numerical results for the mud-flow dam-break experiments by Laigle and Coussot.

\begin{tabular}{lcc}
\hline Variable & Experimental & Computed \\
\hline$\frac{\text { Gauge } 1}{t_{F}[\mathrm{~s}]}$ & 0.48 & \\
$h_{P}[\mathrm{~cm}]$ & 2.56 & 0.58 \\
$h_{R}[\mathrm{~cm}]$ & 1.06 & 2.53 \\
\hline Gauge 2 & & 0.97 \\
\hline$t_{F}[\mathrm{~s}]$ & 1.38 & \\
$h_{P}[\mathrm{~cm}]$ & 2.12 & 1.38 \\
$h_{R}[\mathrm{~cm}]$ & 0.94 & 2.08 \\
\hline Gauge 3 & & 0.97 \\
$t_{F}[\mathrm{~s}]$ & 2.13 & \\
$h_{P}[\mathrm{~cm}]$ & 1.87 & 2.24 \\
$h_{R}[\mathrm{~cm}]$ & 0.69 & 1.73 \\
\hline
\end{tabular}

in which $K$ and $\eta$ are rheological parameters. In the selected experiments $K$ results to be $3.5 \mathrm{~Pa} \cdot \mathrm{s}^{1 / 3}$, while $\eta$ has been empirically set equal to $1 / 3$. Numerical and experimental results are compared in terms of peak height $h_{P}$, residual height $h_{R}$ and front arrival time $t_{F}$ in Table 2, while Figure 6 shows the mixture profile development at different times (a) and computed hydrographs corresponding to 
the three measuring gauges (b). In general peak height is slightly underestimated, while the residual height is sensibly overestimated for gauge 3 . The wave front shows a little delay for gauges 1 and 3, while it is just in time at gauge 2 . However measured hydrographs are generally correctly captured for what concerns their shape, dimension and position in time.
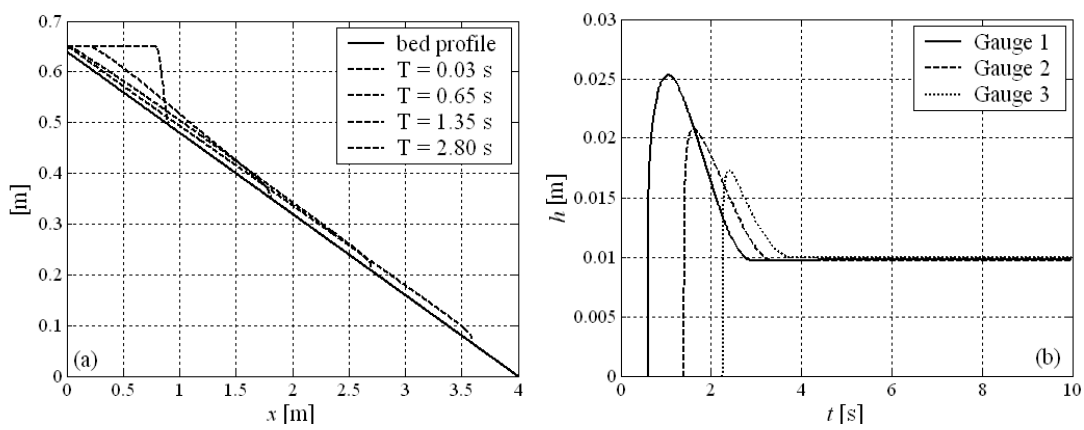

Figure 6: Mud-flow dam-break experiments by Laigle and Coussot: (a) mixture profile evolution in time; (b) flow depth versus time at three measuring gauges.

\section{Conclusions and further developments}

A numerical model for the simulation of dam-break induced mud-flow has been presented. It is based on a mathematical model which main features are concerned with the propagation of the wet-dry fronts, the treatment of irregular and variable cross sections shape, and the applicability to highly sloping channels. Different test cases have been selected in order to verify these features. The classic frictionless dam-break test has been used to test the correctness of waves speed propagation and the capability of treating wet-dry fronts. The source terms treatment has been verified independently, analyzing the influence of pressure terms with a non-cylindrical frictionless ideal channel and the role of friction terms with a mud flow dam-break, for which the analytical solution is available. This test has been performed using different resistance laws, and permitted also to check model flexibility for what concerns the adopted rheological model. Eventually, the model has been tested using laboratory experiments on mud flow dam-break over a sloping plane. Numerical results compare favourably with experiments in terms of front wave speed, peak height and residual front thickness. This test case closes the first phase of model validation, in which all the fundamental features have been investigated and ascertained. The next step could be the extension to debris-flow phenomena and the simulation of a real mud-flow or debris-flow event, to compare numerical results with field data and, since the model has been built for complex geometry channels, to check its behaviour on a real channel configuration. 


\section{References}

[1] Schippa., L. \& Pavan, S., Analytical treatment of source terms for complex channel geometry. Journal of Hydraulic Research, 46(6), pp. 753-763, 2008.

[2] Schippa., L. \& Pavan, S., Bed evolution numerical model for rapidly varying flow in natural streams. Computer \& Geosciences, 35, pp. 390-402, 2009.

[3] Toro, E.F., Riemann Solvers and Numerical Method for Fluid Dynamics, Springer-Verlag Berlin Heidelberg New York, 1999.

[4] Garcia-Navarro, P. \& Vazquez-Cendon M.E., On numerical treatment of the source terms in the shallow water equations. Computer \& Fluids, 29, pp. 951-979, 2000.

[5] Brufau, P., Garcia-Navarro, P., Ghilardi, P., Natale, L. \& Savi, F., 1D Mathematical modelling of debris flow. Journal of Hydraulic Research, 38(6), pp. 435-446, 2000.

[6] Naef, D., Rickenmann, D., Rutschmann, P. \& McArdell, B.W., Comparison of flow resistance relations for debris flow using a one-dimensional finite element simulation model., Natural Hazards and Earth System Sciences, 6, pp.155-165, 2006.

[7] Laigle, D. \& Coussot, P., Numerical modelling of mudflows, Journal of Hydraulic Engineering, 123(7), pp. 617-623, 1997.

[8] Zanuttigh, B. \& Lamberti, A., Analysis of debris wave development with one-dimensional shallow-water equations., Journal of Hydraulic Engineering, 130(4), pp. 293-303, 2004.

[9] Ying, X. \& Wang, S.S.Y., Improved implementation of the HLL approximate Riemann solver for one-dimensional open channel flows. Journal of Hydraulic Research, 46(1), pp. 21-34, 2008.

[10] Goutal, N. \& Maurel, F., Proceedings of the 2nd workshop on dam-break wave simulation, Direction des études et recherches, Electricité de France, Rep. HE43/97/016/B, 1997.

[11] Hungr, O., A model for the runout analysis of rapid flow slides, debris flow, and avalanches. Canadian Geotechnical Journal, 32(4), pp. 610-623, 1995. 